\title{
The symptoms of schizophrenia related to the stability of attractor networks
}

\author{
Marco Loh*1, Edmund T Rolls ${ }^{2}$ and Gustavo Deco ${ }^{1,3}$
}

\author{
Address: ${ }^{1}$ Universitat Pompeu Fabra, Department of Technology, Passeig de Circumval · lació 8, 08003 Barcelona, Spain, ${ }^{2}$ University of Oxford, \\ Department of Experimental Psychology, South Parks Road, Oxford OX1 3UD, UK and ${ }^{3}$ Institució Catalana de Recerca i Estudis Avançats (ICREA), \\ Passeig Lluís Companys, 23, 08010 Barcelona, Spain \\ Email: Marco Loh* - marco.loh@upf.edu \\ * Corresponding author
}

from Sixteenth Annual Computational Neuroscience Meeting: CNS*2007

Toronto, Canada. 7-12 July 2007

Published: 6 July 2007

BMC Neuroscience 2007, 8(Suppl 2):PI42 doi:I0.I I86/I47I-2202-8-S2-PI42

(ㄷ) 2007 Loh et al; licensee BioMed Central Ltd.

We propose a hypothesis to account for the symptoms of schizophrenia in a statistical dynamical systems framework. We propose that the symptoms of schizophrenia are related to alterations in the depth of the basins of attraction, and the statistical effects of spiking neurons, which together influence the stability of cortical attractor neural networks. The cognitive symptoms such as distractibility, working memory deficits and poor attention could be caused by instability of persistent attractor states in prefrontal cortical networks. The negative symptoms, which include a lack of affect and reduction of emotions, may be related to the concomitant decreased firing rates in cortical areas such as the orbitofrontal cortex and anterior cingulate cortex that occur when the depth of the basins of attraction are reduced. The positive symptoms including delusions, paranoia, and hallucinations could arise because the basins of attraction are shallow in temporal lobe semantic memory networks. This would lead thoughts to move too freely around the attractor energy landscape, loosely from thought to weakly associated thought. These hypotheses were investigated in a model of a cortical attractor network based on integrate-and-fire neurons and synaptic currents activated by AMPA, NMDA and GABA receptors [1]. It was found that a decrease in the NMDA receptor conductance leads to a decrease in the stability of working memory states, an increase in distractibility, and lower firing rates, and can potentially account for both the cognitive and negative symptoms. We observed a flat energy landscape associated with the posi- tive symptoms of schizophrenia when, in addition to a reduction in the NMDA conductance, the GABA conductance was reduced. This caused the dynamical system not only to switch between the high firing rate attractor states, but also sometimes to jump from spontaneous activity into one of the attractor states. The findings are consistent with the neurophysiological effects of dopamine, which can influence NMDA and GABA currents. In this approach we thus start with a statistical dynamical systems framework, and use this to help understand how some of the different symptoms of schizophrenia might arise.

\section{Acknowledgements}

ML has been supported by the Boehringer Ingelheim Fonds.

\section{References}

I. Deco G, Rolls ET: Attention, short term memory, and action selection: a unifying theory. Prog Neurobiol 2005, 76:236-256. 